\title{
Salvage chemotherapy with the combination of oxaliplatin, leucovorin, and 5-fluorouracil in advanced gastric cancer refractory or intolerant to fluoropyrimidines, platinum, taxanes, and irinotecan
}

\author{
Chihiro Kondoh ${ }^{1,2}$. Shigenori Kadowaki ${ }^{1}$ Azusa Komori ${ }^{1} \cdot$ Yukiya Narita $^{1} \cdot$ Hiroya Taniguchi $^{1} \cdot$ Takashi Ura $^{1}$. \\ Masashi Ando ${ }^{1} \cdot$ Kei Muro ${ }^{1}$
}

Received: 14 September 2017 / Accepted: 10 April 2018 / Published online: 16 April 2018

(c) The International Gastric Cancer Association and The Japanese Gastric Cancer Association 2018

\begin{abstract}
Background Although nivolumab showed survival benefit in patients with advanced gastric cancer (AGC) progressing after standard chemotherapy, there is a lack of data regarding oxaliplatin-based chemotherapy in this clinical setting.

Methods We retrospectively evaluated the efficacy and safety of oxaliplatin with $l$-leucovorin and bolus/continuous infusion of 5-fluorouracil as salvage treatment in patients with AGC refractory or intolerant to fluoropyrimidines, cisplatin, taxanes, and irinotecan.

Results Overall, 50 patients treated between December 2009 and December 2013 were included in this analysis. The overall response rate (ORR) was $21.2 \%$ among 33 patients with measurable disease. The median time to treatment failure (TTF) and overall survival (OS) were 2.4 and 4.2 months. In multivariate analysis, factors associated with OS included poor performance status [hazard ratio (HR) 3.20; 95\% confidence interval (CI) 1.55-6.60], shorter time from the start of first-line therapy (HR 2.20; 95\% CI 1.18-4.12), and higher neutrophil/lymphocyte ratio value (HR 4.87; 95\% CI 2.32-10.25). In patients $(n=35)$ with at most one risk factor, the ORR, median TTF, and OS were $26.1 \%, 3.6$, and 6.7 months, respectively. The most common grade 3 or 4 adverse events were neutropenia (30\%), anemia (22\%), febrile neutropenia (8\%), and peripheral neuropathy (8\%). Initial and subsequent dose reduction was performed in $18(36 \%)$ and $23(46 \%)$ patients. There was one treatment-related death caused by septic infection.

Conclusions Salvage chemotherapy with the combination of oxaliplatin, leucovorin, and 5-fluorouracil has a potential activity and is tolerable for heavily treated AGC with appropriate dose modification and patient selection.
\end{abstract}

Keywords Gastric cancer · Oxaliplatin $\cdot$ Salvage therapy $\cdot$ Prognostic factor

\section{Introduction}

Systemic chemotherapy in patients with advanced gastric cancer (AGC) leads to improvement of quality of life and prolongation of survival time $[1,2]$. The combination of fluoropyrimidine plus a platinum agent with or without docetaxel or anthracyclines is accepted as a standard therapy

Shigenori Kadowaki

skadowaki@aichi-cc.jp

1 Department of Clinical Oncology, Aichi Cancer Center Hospital, 1-1 Kanokoden, Chikusa-ku, Nagoya, Aichi 464-8681, Japan

2 Department of Medical Oncology, Toranomon Hospital, Toranomon, Minato-ku, Tokyo, Japan with median survival time of 8-14 months [3-8]. Recent randomized prospective trials which compared docetaxel, irinotecan, or ramucirumab monotherapy with the best supportive care in the second- or third-line setting, demonstrated modest gains of approximately 1.5 months for median overall survival (OS) in patients with AGC [9-12]. In addition, a placebo-controlled, randomized phase-III trial (RAIN$\mathrm{BOW}$ ) revealed that ramucirumab, in addition to paclitaxel, improved OS in patients with AGC who progressed during or within 4 months after first-line chemotherapy [13]. Recently, two placebo-controlled phase-III trials of apatinib, an oral small molecule of VEGFR-2 tyrosine kinase inhibitor, and nivolumab blocking the human programmed cell death-1 receptor, showed a survival benefit in patients with AGC progressing after at least two lines of therapy $[14,15]$. In a Japanese phase-III study that compared irinotecan with 
paclitaxel in the second-line setting, $75 \%$ of the patients in the paclitaxel arm received irinotecan as a third-line treatment, resulting in longer OS than that in previous studies [16]. Moreover, three Japanese retrospective studies demonstrated that irinotecan monotherapy has modest activity as third-line chemotherapy for AGC [17-19]. Based on these results, apatinib, nivolumab, or irinotecan monotherapy is a reasonable therapeutic option for selected patients in the third- or later-line setting.

Oxaliplatin is a third generation platinum compound with a spectrum of activity and toxicity different from cisplatin and carboplatin, and has shown a partial lack of cross-resistance with cisplatin and carboplatin, both in vitro and in vivo [20]. The reported response rates of oxaliplatin monotherapy in patients with ovarian cancer refractory to cisplatin or carboplatin ranged from 4.3 to $29.0 \%$ [21, 22]; based on these findings, several studies have investigated the efficacy of oxaliplatin combined with 5-fluorouracil (5-FU) and leucovorin (FOLFOX) in patients with AGC for whom previous chemotherapies had failed [23-28]. The objective response rate was approximately $4.7-26.7 \%$, with the median progression-free survival time (PFS) or time to progression of 2.5-4.3 months and a median OS of 6.2-8.8 months [23-28]. These studies mainly comprised patients treated as second- or third-line treatment, and there are limited data regarding salvage oxaliplatin-based therapy in patients with AGC refractory to 5-FU, cisplatin, taxanes, and irinotecan. The only small retrospective study $(n=12)$ showed that combination therapy with modified FOLFOX-6 (mFOLFOX-6) therapy yielded a response rate of $23.1 \%$, a median PFS of 3.0 months, and a median OS of 8.9 months for this population [28]; however, patients who could derive benefit from salvage oxaliplatin-based chemotherapy remain unclear.

The aim of this study was to assess the safety and efficacy of mFOLFOX-6 in patients with AGC refractory or intolerant to fluoropyrimidine, cisplatin, taxanes and irinotecan, and to explore clinical factors associated with the survival of patients in this clinical setting.

\section{Patients and methods}

\section{Patient population}

Between December 2009 and December 2013, 65 consecutive patients who received mFOLFOX-6 as third- or furtherline therapy at Aichi Cancer Center Hospital were reviewed. Of these, patients with the following criteria were included: (1) histologically proven, inoperable adenocarcinoma of the stomach; (2) an Eastern Cooperative Oncology Group performance status (ECOG PS) score of 0-3; (3) refractory or intolerant to fluoropyrimidines, cisplatin, taxanes, and irinotecan (which could have been given as adjuvant or neoadjuvant chemotherapy or for inoperable disease); and (4) no other combined therapy, including trastuzumab, radiotherapy, or intraperitoneal chemotherapy. As for treatment with adjuvant or neoadjuvant chemotherapy, patients with disease progression during treatment or within 6 months after treatment completion were defined refractory. The collected data were as follows: age, gender, ECOG PS, histological type, previous gastrectomy, number of metastatic sites, metastatic lesions (liver, lymph nodes, or peritoneum), number of prior chemotherapy lines, time from the start of first-line therapy, Glasgow Prognostic Score (GPS), carcinoembryonic antigen (CEA) level, carbohydrate antigen 19-9 (CA19-9) level, neutrophil/lymphocyte ratios (NLR), platelet/lymphocyte ratios (PLR), and time from the start of first-line therapy. Neoadjuvant or adjuvant chemotherapy was considered as prior chemotherapy lines. The GPS was constructed as follows: patients with both an elevated C-reactive protein $(>1.0 \mathrm{mg} / \mathrm{dl})$ and hypoalbuminemia $(<3.5 \mathrm{~g} / \mathrm{dl})$ were allocated a score of 2 , those with only one of these abnormalities were allocated a score of 1 , and those with neither of these abnormalities were allocated a score of 0 . The NLR was defined as the absolute neutrophil count divided by the absolute lymphocyte count; similarly, the PLR was defined as the absolute platelet count divided by the absolute lymphocyte count. All patients signed informed consents prior to receiving oxaliplatin-based chemotherapy. We acquired permission to administer oxaliplatin to heavily pretreated patients in our hospital and submitted a minute description of each patient to the health insurance claims authority until the Japanese insurance system covered this agent in March 2015. The outcome data were last updated on 31 January 2016. This study was approved by the institutional review board of Aichi Cancer Center Hospital.

\section{Treatment and assessment of efficacy and toxicity}

The mFOLFOX-6 therapy consisted of concurrent infusion with oxaliplatin $\left(85 \mathrm{mg} / \mathrm{m}^{2}\right)$ and $l$-leucovorin $\left(200 \mathrm{mg} / \mathrm{m}^{2}\right)$ over a period of $2 \mathrm{~h}$, followed by 5 -FU bolus $\left(400 \mathrm{mg} / \mathrm{m}^{2}\right)$ and immediately continuous $46-\mathrm{h}$ infusion $\left(2400 \mathrm{mg} / \mathrm{m}^{2}\right)$, and the cycle was repeated every 2 weeks. Dose modifications of each antitumor drug, including the initial dose, were decided according to each physician's judgement. Treatment was continued until documented disease progression, unacceptable toxicity, physicians' decision, or patients' refusal. Responses were assessed according to Response Evaluation Criteria in Solid Tumors (RECIST) version 1.1. [29]. Confirmation of the tumor responses was not required for the present study. Toxicity was graded according to National Cancer Institute-Common Toxicity Criteria (NCI-CTC) version 4.0. 


\section{Statistical analysis}

Time to treatment failure (TTF) was defined as the time from the initiation of oxaliplatin-based therapy until treatment discontinuation for any reason or the last follow-up visit. OS was defined as the time from treatment initiation to death from any cause or the last follow-up visit. Time from the start of first-line therapy was calculated from the date of initiation of first-line treatment to the date of the initiation of oxaliplatin-based therapy. The survival curves were estimated using the Kaplan-Meier method and compared using the log-rank test. Uni- and multivariate Cox proportional hazards models were used to identify factors associated with OS, and multivariate analyses were adjusted by relevant variables with $p$ values of $<0.20$ in univariate analyses. The cutoff for NLR and PLR utilized to discriminate between better survival (median OS, $\geq 6$ months) and poor survival was determined based on the result of the receiver operating characteristic (ROC) curve analysis [30]. All statistical analyses were performed using Dr. SPSS II software (SPSS Japan Inc., Tokyo, Japan) and were two-sided, and statistical significance was defined as $p$ values of $<0.05$.

\section{Results}

\section{Patient characteristics}

A total of 50 patients were eligible for the study. The background characteristics are listed in Table 1. The median age of the patients was 67 years (range 36-78 years). Sixteen patients (28\%) had an ECOG PS of 2 or 3 . The dominant histological type was poorly differentiated adenocarcinoma, and $38(76 \%)$ patients had peritoneal metastasis. Only four (8\%) patients presented with human epidermal growth factor receptor 2 (HER2)-positive tumors, two (4\%) patients received trastuzumab as the first-line treatment, and the remaining patients received trastuzumab as the third-line or fifth-line treatment before mFOLFOX-6 therapy. The number of prior chemotherapy lines was 2 or 3 in 35 (70\%), 4 in $9(18 \%)$, and 5 or more in six $(12 \%)$ patients. All the patients had previously received cisplatin, fluoropyrimidines, and taxanes, and 43 (86\%) had prior irinotecan administration. Seven patients with risk factors, such as symptomatic gastrointestinal obstruction and massive ascites, did not receive irinotecan. Median time from the start of first-line therapy was 19.2 months (range 2.3-46.4 months).

\section{Tumor response and survival}

At the time of analysis, all but one patient with lost follow-up data had disease progression and had died from disease progression. The clinical outcomes according to
Table 1 Patient characteristics

\begin{tabular}{|c|c|c|}
\hline Characteristics $(n=50)$ & No. of patients & $\%$ \\
\hline \multicolumn{3}{|l|}{ Age, years } \\
\hline Median (range) & $67(36-78)$ & - \\
\hline \multicolumn{3}{|l|}{ Gender } \\
\hline Male/female & $29 / 21$ & $58 / 42$ \\
\hline \multicolumn{3}{|l|}{ Performance status } \\
\hline $0 / 1 / 2 / 3$ & $10 / 26 / 8 / 6$ & $20 / 52 / 16 / 12$ \\
\hline \multicolumn{3}{|l|}{ Histological type } \\
\hline Differentiated/undifferentiated & $12 / 38$ & $24 / 76$ \\
\hline \multicolumn{3}{|l|}{ Gastrectomy } \\
\hline Yes/no & $29 / 21$ & $58 / 42$ \\
\hline \multicolumn{3}{|c|}{ Neoadjuvant/adjuvant chemotherapy } \\
\hline Yes/no & $26 / 24$ & $52 / 48$ \\
\hline \multicolumn{3}{|l|}{ Number of metastasis } \\
\hline $1 / 2 / \geq 3$ & $20 / 17 / 13$ & $40 / 34 / 26$ \\
\hline \multicolumn{3}{|l|}{ Metastatic sites* } \\
\hline Peritoneum/lymph node/liver & $39 / 27 / 16$ & $78 / 54 / 32$ \\
\hline \multicolumn{3}{|l|}{ Number of prior chemotherapy lines } \\
\hline $2 / 3 / \geq 4$ & $12 / 23 / 15$ & $24 / 46 / 30$ \\
\hline \multicolumn{3}{|c|}{ Time from the start of first-line therapy } \\
\hline Median (range) & $19.2(2.3-46.4)$ & - \\
\hline \multicolumn{3}{|l|}{ Glasgow prognostic score } \\
\hline 0/1/2/unknown & $15 / 15 / 18 / 2$ & $30 / 30 / 36 / 4$ \\
\hline \multicolumn{3}{|l|}{ Serum CEA, ng/ml } \\
\hline Median (range) & $4.7(1.2-14,694)$ & - \\
\hline \multicolumn{3}{|l|}{ Serum CA19-9, U/ml } \\
\hline Median (range) & $24.3(0.1-175,900)$ & - \\
\hline \multicolumn{3}{|l|}{ Neutrophil lymphocyte ratio } \\
\hline Median (range) & $3.0(1.2-195)$ & - \\
\hline \multicolumn{3}{|l|}{ Platelet lymphocyte ratio } \\
\hline Median (range) & $243.1(40.0-3076)$ & - \\
\hline
\end{tabular}

CEA carcinoembryonic antigen, CA19-9 carbohydrate antigen 19-9

*Overlapped data

each chemotherapeutic regimen are shown in Table 2 . In all, $33(66 \%)$ patients had measurable lesions based on the RECIST criteria. Among them, no complete response and seven partial responses were achieved, for the overall response rate (ORR) of $21.2 \%$ [95\% confidence interval (CI) 6.5-35.9\%]. Among all the patients, the disease control rate was $48.0 \%$ (95\% CI 33.7-62.6\%). The median TTF was 2.4 months (95\% CI 1.9-2.9 months; Fig. 1a) and the median OS was 4.2 months (95\% CI 2.4-6.0 months; Fig. 1b). Thirteen (26\%) patients received subsequent systemic chemotherapy; taxanes were given in seven patients, investigational new drugs in four, and others in two. Ramucirumab and immune-checkpoint inhibitors, including nivolumab, were not administered in any treatment line. 
Table 2 Activity of mFOLFOX-6 therapy

\begin{tabular}{lll}
\hline & $n=50$ & $\%$ \\
\hline $\begin{array}{l}\text { Measurable disease } \\
\text { Tumor response }\end{array}$ & $n=33$ & \\
Complete response & 0 & 0 \\
Partial response & 7 & 21.2 \\
Stable disease & 9 & 27.3 \\
Progressive disease & 9 & 27.3 \\
Not evaluable & 8 & 24.2 \\
Response rate, $\%$ & & 21.2 \\
& & $(95 \%$ CI \\
& & $6.5-35.9)$ \\
\hline
\end{tabular}

$m F O L F O X-6$ bolus and infused 5-fluorouracil plus $l$-leucovorin plus oxaliplatin, $C I$ confidence interval

\section{Toxicity and treatment exposure}

The median number of treatment cycles was 3.5 (range 1-20). The toxicity profiles are listed in Table 3 . The most common grade $3 / 4$ hematological toxicity was neutropenia (30\%), and grade 3 febrile neutropenia was observed in four (8\%) patients. The common nonhematologic toxicity was anorexia (66\%), fatigue (52\%), peripheral neuropathy (42\%), and nausea $(46 \%)$, but most of them were manageable. Grade 3 peripheral neuropathy was observed in four (8\%) patients, for which oxaliplatin was discontinued. Only one (2\%) patient with an ECOG PS of 3 died from septic shock due to bacterial translocation during mFOLFOX-6 therapy, and this event was considered treatment-related. Five (10\%) patients died due to disease progression within 30 days of the last dosage of therapy.

Initial dose and a subsequent dose reduction were performed in 18 (36\%) and 23 (46\%) patients. Relative dose intensity, defined as the proportion actually received of the reference standard dose intensity for each regimen, was at a mean of $75.0 \%$ (range $22.4-100 \%$ ), $82.4 \%$ (range $37.3-100 \%$ ), and $56.8 \%$ (range $0-100 \%$ ) for oxaliplatin, 5-FU continuous infusion, and 5-FU bolus infusion, respectively. Mean values of relative dose intensity for each regimen were $73.6,81.8$, and $58.1 \%$ for oxaliplatin, 5-FU continuous infusion, and 5-FU bolus infusion, respectively, only among patients with an ECOG PS of 0-2.

The treatment was discontinued in all patients. The reasons for discontinuation were disease progression in 43 $(86 \%)$ patients, unacceptable toxicity in four (8\%), patient's refusal in two (4\%), and treatment-related death in one (2\%).

\section{Clinical factors associated with OS}

The results of uni- and multivariate analyses for OS are presented in Table 4. The cutoff PLR and NLR levels for predicting OS of $\geq 6$ months were 305 and 3.5 , respectively,

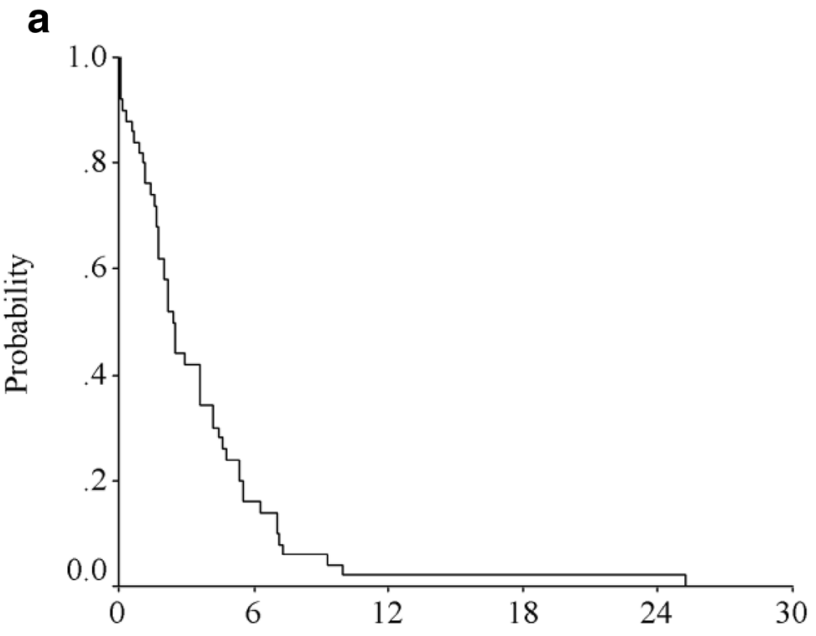

Time (months)
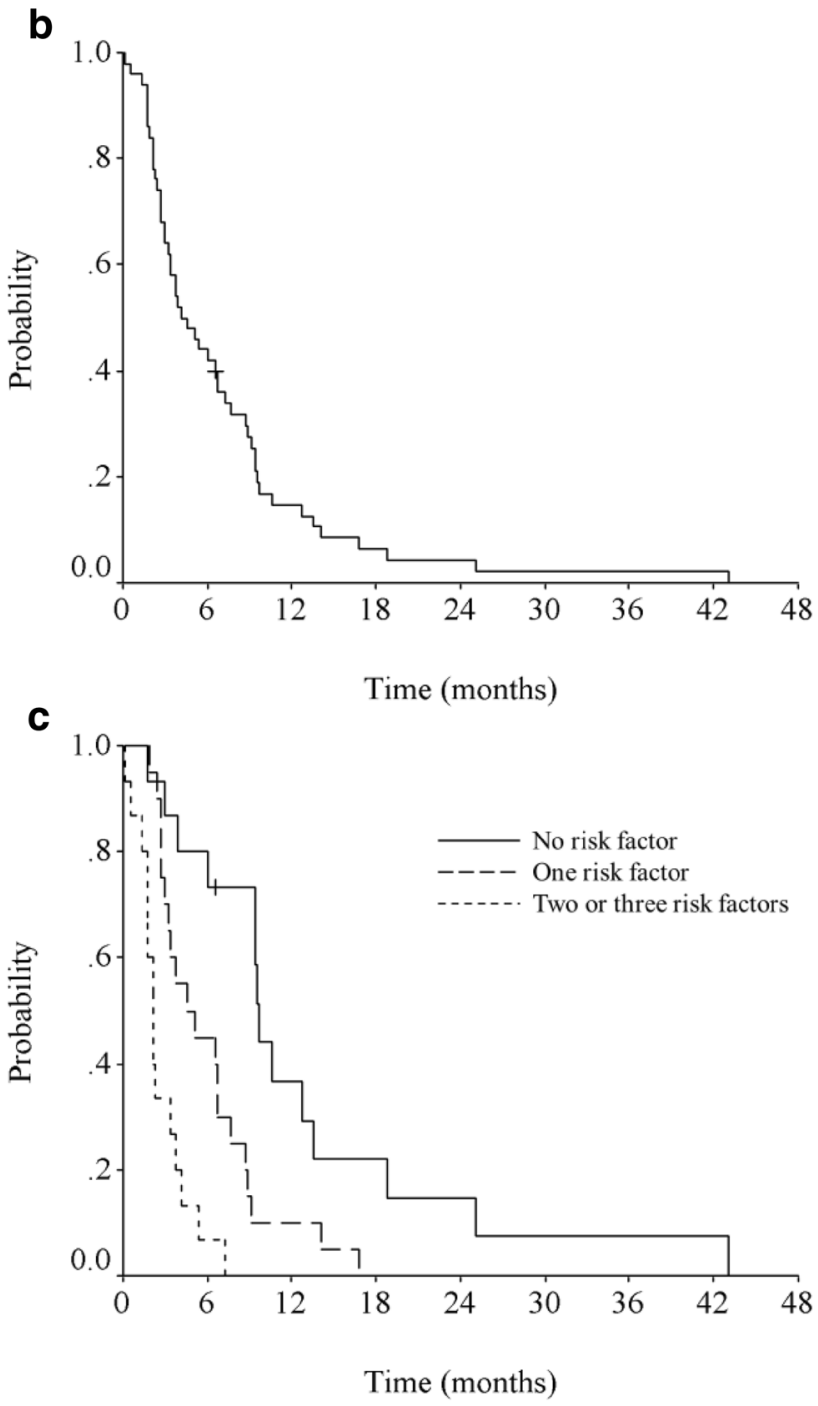

Fig. 1 Kaplan-Meier curves of time to treatment failure and overall survival. a survival curve of time to treatment failure. $\mathbf{b}$ survival curve of overall survival. c survival curve of overall survival according to the number of prognostic factors 
Table 3 Toxicities according to the NCI-CTC grade (version 4.0)

\begin{tabular}{lcc}
\hline Type of toxicity & $n=50$ & \\
\cline { 2 - 3 } & All & $\mathrm{Gr} \geq 3(\%)$ \\
\hline Hematologic toxicity & 27 & $15(30)$ \\
Neutropenia & 30 & $11(22)$ \\
Anemia & 27 & $1(2)$ \\
Thrombocytopenia & & \\
Non-hematologic toxicity & 33 & $0(0)$ \\
Anorexia & 23 & $0(0)$ \\
Nausea & 7 & $1(2)$ \\
Vomiting & 26 & $0(0)$ \\
Fatigue & 9 & $0(0)$ \\
Diarrhea & 6 & $0(0)$ \\
Mucositis & 4 & $1(2)$ \\
Allergic reaction & 21 & $4(8)$ \\
Peripheral neuropathy & 4 & $4(8)$ \\
Febrile neutropenia & & \\
\hline
\end{tabular}

$m F O L F O X-6$ bolus and infused 5-fluorouracil plus $l$-leucovorin plus oxaliplatin

and the areas under the ROC curve were 0.66 (95\% CI $0.50-0.82 ; p=0.07$ ) and 0.73 (95\% CI $0.57-0.88 ; p=0.01$ ), respectively. Based on these results, the cutoff levels for the PLR and NLR were set at 300 and 3.5 in the current study. In the univariate analysis, being female, an ECOG PS of 2 or 3 , no previous gastrectomy, time from the start of therapy of $<18$ months, a GPS of 2 , a NLR of $\geq 3.5$, and a PLR of $\geq 300$ were significant predictors of worse OS. Multivariate analysis identified the following three prognostic factors: ECOG PS of 2 or 3 (HR 3.20, 95\% CI 1.55-6.60; $p=0.002$ ), time from the start of first-line therapy of $<18$ months (HR $2.20,95 \%$ CI $1.18-4.12 ; p=0.014$ ), and a NLR of $\geq 3.5$ (HR $4.87,95 \%$ CI $2.32-10.25 ; p<0.001)$.

Median OS in patients with no prognostic factor $(n=15)$, one factor $(n=20)$, and two or three factors $(n=15)$ were 9.7 months (95\% CI 9.2-10.2), 4.6 months (95\% CI 1.8-7.4), and 2.2 months (95\% CI 1.7-2.7), respectively (Fig. 1c). There was a significant difference in OS among these three risk groups (log-rank $p<0.001)$. The ORR and the disease control rate for each group were 27\% (3 of 11) and 73\% (11 of 15 ), $25 \%$ ( 3 of 12 ) and $45 \%$ ( 9 of 20 ), and $10 \%$ ( 1 of 10 ), and $27 \%$ (4 of 15$)$.

\section{Discussion}

There have been few data regarding salvage treatment for AGC refractory or intolerant to fluoropyrimidines, cisplatin, taxanes, and irinotecan. In this study, we evaluated the efficacy and safety of mFOLFOX-6 for heavily treated patients refractory or intolerant to standard chemotherapy, with a response rate of $21 \%$, and median TTF and OS of 2.4 and 4.2 months, respectively. Observed toxicities were generally feasible. In addition, our study assessed factors to
Table 4 Factors associated with overall survival

\begin{tabular}{|c|c|c|c|c|}
\hline \multirow[t]{2}{*}{ Variables } & \multicolumn{2}{|l|}{ Univariate } & \multicolumn{2}{|l|}{ Multivariate } \\
\hline & HR $(95 \%$ CI $)$ & $p$ value & HR $(95 \%$ CI $)$ & $p$ value \\
\hline Age $(\geq 65$ vs. $<65)$ & $1.12(0.63-1.98)$ & 0.70 & & \\
\hline Gender (female vs. male) & $2.05(1.14-3.70)$ & 0.02 & & \\
\hline Performance status ( $\geq 2$ vs. $0-1$ ) & $2.61(1.33-5.13)$ & 0.005 & $3.20(1.55-6.60)$ & 0.002 \\
\hline Differentiation (undiff. vs. diff.) & $1.89(0.91-3.96)$ & 0.09 & & \\
\hline Gastrectomy (no vs. yes) & $1.89(1.03-3.47)$ & 0.04 & & \\
\hline \multicolumn{5}{|l|}{ Metastatic sites (yes vs. no) } \\
\hline Liver & $1.01(0.55-1.85)$ & 0.98 & & \\
\hline Lymph node & $0.87(0.48-1.55)$ & 0.63 & & \\
\hline Peritoneum & $1.66(0.81-3.37)$ & 0.16 & & \\
\hline No. of metastatic sites ( $\geq 3$ vs. $0-2$ ) & $1.17(0.61-2.22)$ & 0.64 & & \\
\hline $\begin{array}{l}\text { Time from the start of therapy, months } \\
(<18 \text { vs. } \geq 18)\end{array}$ & $2.47(1.36-4.46)$ & 0.003 & $2.20(1.18-4.12)$ & 0.014 \\
\hline CEA, ng/ml $(\geq 50$ vs. $<50)$ & $1.39(0.70-2.77)$ & 0.35 & & \\
\hline CA19-9, U/ml ( $\geq 38$ vs. $<38)$ & $0.90(0.50-1.63)$ & 0.73 & & \\
\hline GPS (2 vs. $0-1)$ & $2.13(1.16-3.91)$ & 0.02 & & \\
\hline $\operatorname{NLR}(\geq 3.5$ vs. $<3.5)$ & $4.07(2.01-8.04)$ & $<0.001$ & $4.87(2.32-10.25)$ & $<0.001$ \\
\hline PLR $(\geq 300$ vs. $<300)$ & $1.60(0.88-2.93)$ & 0.13 & & \\
\hline
\end{tabular}

$H R$ hazard ratio, $C I$ confidence interval, Undiff. undifferentiated, Diff. differentiated, CEA carcinoembryonic antigen, CA19-9 carbohydrate antigen 19-9, NLR neutrophil lymphocyte ratio, PLR platelet lymphocyte ratio 
predict patients who are likely to benefit from oxaliplatinbased chemotherapy, and higher pre-treatment NLR levels, shorter time from the initiation of first-line therapy, and poor PS were negative clinical indicators for OS, after adjustment for other relevant factors.

Based on two recent phase-III trials, third- or further-line therapy with apatinib or nivolumab prolongs the survival of selected patients with a good PS (0 or 1) progressing after standard chemotherapy $[14,15]$. The reported ORR, median PFS, and OS were $2.8 \%, 2.6$, and 6.5 months for apatinib and $11.2 \%, 1.6$ months, and 5.3 months for nivolumab. As for treatment lines, $34 \%$ of patients in the apatinib group and $79 \%$ of patients in the nivolumab group were treated as fourth- or further-line therapy. In recent retrospective studies, the ORR and median PFS of irinotecan and ramucirumab monotherapy as third- or further-treatment line have been reported to be $3-18$ and $7.7 \%$, and $2.2-3.8$ and 2.1 months, respectively [17-19, 31]. In the present study, patients were expected to have worse outcomes than the patient population in the phase-III studies of apatinib and nivolumab (PS 2 or 3 in $28 \%$ and third or more lines of therapy fail in $76 \%$ ), but comparable survival and a better response rate were achieved than those of apatinib, nivolumab, irinotecan, and ramucirumab. In the salvage setting, there is marked heterogeneity in the duration of survival among patients and it is frequently at the expense of toxicity for this population. Therefore, the selection of patients who are more likely to benefit from salvage treatment is an important issue. There are a few studies addressing this issue in the salvage setting: in a retrospective study and one phase 2 study from Korea, PS was the only independent prognostic factor in OS [26, 27]. ECOG PS has been reported to be the universal prognostic factor in any line of treatment and in various malignancies, and was a reproducible marker predicting survival in the present study as well. In the third-line setting, shorter duration from first-line to third-line therapy has been reported to be correlated with a worse prognosis [18, 32], corresponding to our results. Inflammation plays a critical role in the progression of many cancers, by promoting cancer-cell proliferation, angiogenesis, and metastasis, and by impacting tumor response to chemotherapy. In early line therapy settings, the laboratory index of systematic inflammatory response including GPS [33], NLR [34, 35], and PLR [35] have been investigated as prognostic and predictive markers in patients with AGC, and these were negatively correlated with survival outcome. Although these are easily calculated, reproducible, and inexpensive markers of systemic inflammation, their significance in later-line therapy setting has not been studied fully. For the first time, we investigated the significance of GPS, NLR, and PNR in a salvage setting. Among these, only NLR was a prognostic indicator of OS in accordance with previous studies, but this can be attributed to the small sample size in the present study. Hence, further investigation is needed. A high NLR reflects both a heightened neutrophil-dependent inflammatory reaction and a decreased lymphocyte-mediated antitumor response, which contribute to cancer progression and poor prognosis. The median value of NLR in our cohort was higher than that from previous studies, reflecting the far advanced disease status of patients included in our study. When confined to patients with two or three prognostic markers identified, the response rate, median TTF, and OS were 10\%, 1.4, and 2.2 months, respectively, indicating that this patient population is not a candidate population for salvage oxaliplatin-based therapy.

With regards to toxicity, mFOLFOX-6 therapy was totally feasible. The most common grade $3 / 4$ hematological toxicity was neutropenia (30\%) with $8 \%$ of febrile neutropenia, which was comparable to the incidence (17.9-44.6 and 0.2-19.2\%, respectively) observed in previous studies [23-28]. The most common non-hematological toxicities included anorexia (66\%), fatigue (52\%), peripheral neuropathy (42\%), and diarrhea (18\%). These toxicities were acceptable in terms of incidence and severity, but clinically significant neuropathy developed in $8 \%$ of patients. A possible reason for the relatively frequent incidence of neuropathy compared to that $(0-3.8 \%)$ in previous studies could be that all patients were heavily pretreated with neurotoxic agents such as cisplatin and taxanes. A 75-year-old female patient who experienced treatment-related death had an ECOG PS of 3 and essentially should have received the best supportive care. Generally, the toxicity profile and its incidence in our study did not show marked differences compared with previous reports, but our results should be interpreted with caution because initial and/or subsequent dose reduction was performed in 36 and $46 \%$ of the patients. We should carefully select patients who may benefit from the salvage chemotherapy in terms of the safety of such treatment as well.

Our study has some limitations. First, this is a retrospective study of a widely varied patient population in a single institute. Second, the total number of patients analyzed is relatively small. Accordingly, other factors that were not found to be significantly associated with outcome in the present study might have been important in a larger patient cohort. Finally, our findings are not validated; therefore, external validation of our results is required in larger patient cohorts. Despite these limitations, our observation strengthens demands for further investigation of mFOLFOX-6 as salvage treatment in patients with AGC.

In conclusion, our results indicate that salvage chemotherapy with mFOLFOX-6 regimen had modest efficacy with an acceptable toxic profile in patients with AGC heavily treated with standard chemotherapy. Patients with at most one risk factor such as poor PS, high NLR, or short time from the initiation of first-line therapy might be good candidates to derive clinical benefit from these regimens. 
Acknowledgements The authors would like to thank Enago (www. enago.jp) for the English language review.

\section{Compliance with ethical standards}

Conflict of interest None of the authors have financial or personal conflicts of interest to disclose.

Human rights statement and informed consent All procedures followed were in accordance with the ethical standards of the responsible committee on human experimentation (institutional and national) and with the Helsinki Declaration of 1964 and later versions. Informed consent or a substitute for it was obtained from all patients for being included in the study.

\section{References}

1. Glimelius B, Ekstrom K, Hoffman K, Graf W, Sjoden PO, Haglund $U$, et al. Randomized comparison between chemotherapy plus best supportive care with best supportive care in advanced gastric cancer. Ann Oncol. 1997;8:163-8.

2. Murad AM, Santiago FF, Petroianu A, Rocha PR, Rodrigues MA, Rausch M. Modified therapy with 5-fluorouracil, doxorubicin, and methotrexate in advanced gastric cancer. Cancer. 1993;72:37-41.

3. Van Cutsem E, Moiseyenko VM, Tjulandin S, Majlis A, Constenla $\mathrm{M}$, Boni $\mathrm{C}$, et al. Phase III study of docetaxel and cisplatin plus fluorouracil compared with cisplatin and fluorouracil as first-line therapy for advanced gastric cancer: a report of the V325 Study Group. J Clin Oncol. 2006;24:4991-7.

4. Cunningham D, Starling N, Rao S, Iveson T, Nicolson M, Coxon F, et al. Capecitabine and oxaliplatin for advanced esophagogastric cancer. N Engl J Med. 2008;358:36-46.

5. Kang YK, Kang WK, Shin DB, Chen J, Xiong J, Wang J, et al. Capecitabine/cisplatin versus 5-fluorouracil/cisplatin as first-line therapy in patients with advanced gastric cancer: a randomised phase III noninferiority trial. Ann Oncol. 2009;20:666-73.

6. Koizumi W, Narahara H, Hara T, Takagane A, Akiya T, Takagi M, et al. S-1 plus cisplatin versus S-1 alone for first-line treatment of advanced gastric cancer (SPIRITS trial): a phase III trial. Lancet Oncol. 2008;9:215-21.

7. Al-Batran SE, Hartmann JT, Probst S, Schmalenberg H, Hollerbach S, Hofheinz R, et al. Phase III trial in metastatic gastroesophageal adenocarcinoma with fluorouracil, leucovorin plus either oxaliplatin or cisplatin: a study of the Arbeitsgemeinschaft Internistische Onkologie. J Clin Oncol. 2008;26:1435-42.

8. Yamada Y, Higuchi K, Nishikawa K, Gotoh M, Fuse N, Sugimoto N, et al. Phase III study comparing oxaliplatin plus S-1 with cisplatin plus S-1 in chemotherapy-naive patients with advanced gastric cancer. Ann Oncol. 2015;26:141-8.

9. Fuchs CS, Tomasek J, Yong CJ, Dumitru F, Passalacqua R, Goswami $\mathrm{C}$, et al. Ramucirumab monotherapy for previously treated advanced gastric or gastro-oesophageal junction adenocarcinoma (REGARD): an international, randomised, multicentre, placebocontrolled, phase 3 trial. Lancet. 2014;383:31-9.

10. Ford HE, Marshall A, Bridgewater JA, Janowitz T, Coxon FY, Wadsley J, et al. Docetaxel versus active symptom control for refractory oesophagogastric adenocarcinoma (COUGAR-02): an open-label, phase 3 randomised controlled trial. Lancet Oncol. 2014;15:78-86.

11. Kang JH, Lee SI, Lim do H, Park KW, Oh SY, Kwon HC, et al. Salvage chemotherapy for pretreated gastric cancer: a randomized phase III trial comparing chemotherapy plus best supportive care with best supportive care alone. J Clin Oncol. 2012;30:1513-8.

12. Thuss-Patience PC, Kretzschmar A, Bichev D, Deist T, Hinke A, Breithaupt K, et al. Survival advantage for irinotecan versus best supportive care as second-line chemotherapy in gastric cancer-a randomised phase III study of the Arbeitsgemeinschaft Internistische Onkologie (AIO). Eur J Cancer. 2011;47:2306-14.

13. Wilke H, Muro K, Van Cutsem E, Oh SC, Bodoky G, Shimada Y, et al. Ramucirumab plus paclitaxel versus placebo plus paclitaxel in patients with previously treated advanced gastric or gastrooesophageal junction adenocarcinoma (RAINBOW): a doubleblind, randomised phase 3 trial. Lancet Oncol. 2014;15:1224-35.

14. Li J, Qin S, Xu J, Xiong J, Wu C, Bai Y, et al. Randomized, double-blind, placebo-controlled phase III trial of apatinib in patients with chemotherapy-refractory advanced or metastatic adenocarcinoma of the stomach or gastroesophageal junction. J Clin Oncol. 2016;34:1448-54.

15. Kang Y-H, Satoh T, Ryu M-H, Chao Y, Kato K, Chung H-C, et al. Nivolumab (ONO-4538/BMS-936558) as salvage treatment after second or later-line chemotherapy for advanced gastric or gastro-esophageal junction cancer (AGC): A double-blinded, randomized, phase III trial. J Clin Oncol. 2017;35:4s. (suppl; abstract 2).

16. Hironaka S, Ueda S, Yasui H, Nishina T, Tsuda M, Tsumura T, et al. Randomized, open-label, phase III study comparing irinotecan with paclitaxel in patients with advanced gastric cancer without severe peritoneal metastasis after failure of prior combination chemotherapy using fluoropyrimidine plus platinum: WJOG 4007 trial. J Clin Oncol. 2013;31:4438-44.

17. Kawakami T, Machida N, Yasui H, Kawahira M, Kawai S, Kito Y, et al. Efficacy and safety of irinotecan monotherapy as third-line treatment for advanced gastric cancer. Cancer Chemother Pharmacol. 2016;78:809-14.

18. Nishimura T, Iwasa S, Nagashima K, Okita N, Takashima A, Honma Y, et al. Irinotecan monotherapy as third-line treatment for advanced gastric cancer refractory to fluoropyrimidines, platinum, and taxanes. Gastric Cancer. 2017;20:655-62.

19. Makiyama A, Arimizu K, Hirano G, Makiyama C, Matsushita Y, Shirakawa $\mathrm{T}$, et al. Irinotecan monotherapy as third-line or later treatment in advanced gastric cancer. Gastric Cancer. 2017. https ://doi.org/10.1007/s10120-017-0759-9.

20. Pendyala L, Creaven PJ. In vitro cytotoxicity, protein binding, red blood cell partitioning, and biotransformation of oxaliplatin. Cancer Res. 1993;53:5970-6.

21. Fracasso PM, Blessing JA, Morgan MA, Sood AK, Hoffman JS. Phase II study of oxaliplatin in platinum-resistant and refractory ovarian cancer: a gynecologic group study. J Clin Oncol. 2003;21:2856-9.

22. Chollet P, Bensmaine MA, Brienza S, Deloche C, Cure H, Caillet $\mathrm{H}$, et al. Single agent activity of oxaliplatin in heavily pretreated advanced epithelial ovarian cancer. Ann Oncol. 1996;7:1065-70.

23. Kim DY, Kim JH, Lee SH, Kim TY, Heo DS, Bang YJ, et al. Phase II study of oxaliplatin, 5-fluorouracil and leucovorin in previously platinum-treated patients with advanced gastric cancer. Ann Oncol. 2003;14:383-7.

24. Suh SH, Kwon HC, Jo JH, Cho YR, Seo BG, Lee DM, et al. Oxaliplatin with biweekly low dose leucovorin and bolus and continuous infusion of 5-fluorouracil (modified FOLFOX 4) as a salvage therapy for patients with advanced gastric cancer. Cancer Res Treat. 2005;37:279-83.

25. Jeong J, Jeung HC, Rha SY, Im CK, Shin SJ, Ahn JB, et al. Phase II study of combination chemotherapy of 5-fluorouracil, lowdose leucovorin, and oxaliplatin (FLOX regimen) in pretreated advanced gastric cancer. Ann Oncol. 2008;19:1135-40.

26. Seo HY, Kim DS, Choi YS, Sung HJ, Park KH, Choi IK, et al. Treatment outcomes of oxaliplatin, 5-FU, and leucovorin as 
salvage therapy for patients with advanced or metastatic gastric cancer: a retrospective analysis. Cancer Chemother Pharmacol. 2009;63:433-9.

27. Kim YS, Hong J, Sym SJ, Park SH, Park J, Cho EK, et al. Oxaliplatin, 5-fluorouracil and leucovorin (FOLFOX-4) combination chemotherapy as a salvage treatment in advanced gastric cancer. Cancer Res Treat. 2010;42:24-9.

28. Tsuji K, Yasui H, Onozawa Y, Boku N, Doyama H, Fukutomi A, et al. Modified FOLFOX-6 therapy for heavily pretreated advanced gastric cancer refractory to fluorouracil, irinotecan, cisplatin and taxanes: a retrospective study. Jpn J Clin Oncol. 2012;42:686-90.

29. Eisenhauer EA, Therasse P, Bogaerts J, Schwartz LH, Sargent D, Ford R, et al. New response evaluation criteria in solid tumours: revised RECIST guideline (version 1.1). Eur J Cancer. 2009;45:228-47.

30. Youden WJ. Index for rating diagnostic tests. Cancer. 1950;3:32-5.

31. Murahashi S, Takahari D, Wakatsuki T, Fukuda N, Ichimura T, Ogura M. A retrospective analysis of ramucirumab monotherapy in previously treated Japanese patients with advanced or metastatic gastric adenocarcinoma. Int J Clin Oncol. 2017. https ://doi.org/10.1007/s10147-017-1192-0.

32. Kang EJ, Im SA, Oh DY, Han SW, Kim JS, Choi IS, et al. Irinotecan combined with 5-fluorouracil and leucovorin third-line chemotherapy after failure of fluoropyrimidine, platinum, and taxane in gastric cancer: treatment outcomes and a prognostic model to predict survival. Gastric Cancer. 2013;16:581-9.

33. Crumley AB, McMillan DC, McKernan M, McDonald AC, Stuart RC. Evaluation of an inflammation-based prognostic score in patients with inoperable gastro-oesophageal cancer. Br J Cancer. 2006;94:637-41.

34. Yamanaka T, Matsumoto S, Teramukai S, Ishiwata R, Nagai Y, Fukushima M. The baseline ratio of neutrophils to lymphocytes is associated with patient prognosis in advanced gastric cancer. Oncology. 2007;73:215-20.

35. Lee S, Oh SY, Kim SH, Lee JH, Kim MC, Kim KH, et al. Prognostic significance of neutrophil lymphocyte ratio and platelet lymphocyte ratio in advanced gastric cancer patients treated with FOLFOX chemotherapy. BMC Cancer. 2013;13:350. 